\title{
Dislocations as origin of high critical current density in bulk $\mathrm{MgB}_{2}$
}

\author{
Pavlo Mikheenko \\ Department of Physics \\ University of Oslo \\ Oslo, Norway \\ pavlo.mikheenko@fys.uio.no
}

\begin{abstract}
MgB}_{2}$ is an important superconducting material that could be used as a basis of liquid-hydrogen renewable energy economy. The distinctive property of this material is its very high critical current density in bulk reaching $10^{6} \mathrm{~A} / \mathrm{cm}^{2}$. It is found that such a high critical current density is of nanoscale origin, and is linked to the pinning of magnetic flux quanta on grain boundaries. To advance this knowledge, a combination of atomic force microscopy, electron backscattered diffraction and SQUID magnetometry was used to clarify the exact dependence of critical current density on the density of dislocations in a cross-section of dense polycrystalline samples. It is found that in a system of randomly oriented grains, the dislocations residing in twist grain boundaries and their cross-points are responsible for the strong pinning in the material.
\end{abstract}

Keywords-superconductivity, nanostructure, $\mathrm{MgB} 2$, grain boundaries, dislocations, pinning, critical current density.

\section{INTRODUCTION}

In spite of recent success in increasing critical temperature in superconducting materials, see for example [1], one specific compound with modest critical temperature of $39 \mathrm{~K}$, namely $\mathrm{MgB}_{2}$ remains in the center of research activity. The reason is that it demonstrates a very high density of critical current $\left(J_{c}\right)$ in the bulk at boiling temperature of liquid hydrogen $(20 \mathrm{~K})$. At this temperature, $J_{c}$ reaches $10^{6} \mathrm{~A} / \mathrm{cm}^{2}$, some three order of magnitude higher than in bulk high temperature superconductors. There have been many attempts to clarify origin of high $J_{c}$ or, related to it, strong pinning in $\mathrm{MgB}_{2}$. Several pinning mechanisms were identified, however main object responsible for high $J_{c}$ remains elusive. The reason is that while $J_{c}$ is a property of material on a macroscopic scale, individual pinning forces act on the nanometer scale. Such distances are typically outside of the reach of traditional instruments at low temperatures, and the integration of the individual forces allowing predicting $J_{c}$ is not a trivial issue. A specific obstacle is high porosity and poor connectivity between the grains in conventionally sintered $\mathrm{MgB}_{2}$, which is the main source of variation in its local properties. The latter does not allow designing experiments, in which the dominant change in $J_{c}$ comes from the variation of the (controlled) number of pinning centers. Here this obstacle was overcome by preparing a large number of dense samples.

A traditional method to increase the number of pinning centers is the addition of nanoparticles. A vast number of publications demonstrates improvement of $J_{c}$ by this technique. The most effective nanoparticle appears to be $\mathrm{SiC}$ [2]. However, its partially beneficial effect is due to enhancement of second critical field caused by the diffusion of carbon. Although it was concluded in [2] that small grain size and nano-inclusions increase $J_{c}$, no specific defects of crystal lattice were identified, which could be responsible for the improvement in pinning. Moreover, nanoparticles tend to decrease $J_{c}$ in low magnetic fields, indicating reduction in the cross-section for supercurrent and degrading (in contrast to the low additions of carbon) modification of $\mathrm{MgB}_{2}$ by the diffusion of elements. The important questions are how to reach the balance between positive and negative effects when modifying pinning by nanoparticles and whether strong pinning could be achieved without nanoparticles at all, just by structural modifications. To clarify this, here two groups of dense samples has been measured. One was without nanoparticles (11 samples) and one with nanoparticles (14 samples). Most of the samples and with the same types of nanoparticles were as in [3].

\section{EXPERIMENTAL}

\section{A. Samples preparation}

The dense samples were prepared by two advanced methods: hot isostatic pressing (HIP) and resistive sintering (RS). In HIP, a pre-compacted $\mathrm{MgB}_{2}$ powder is enclosed in a steel container, evacuated and pressed at $1 \mathrm{kbar}$ in an argon chamber at temperature of $\sim 1000{ }^{\circ} \mathrm{C}$. In $\mathrm{RS}$, powder is uniaxially pressed in vacuum in a graphite die with tungsten rods carrying electrical current up to $1000 \mathrm{~A}$. The electrical current heats the powder above $800{ }^{\circ} \mathrm{C}$. More details on the preparation methods are available in [3].

\section{B. Measurements techniques}

To access critical current, magnetization loops were recorded on small (typically $0.6 \times 0.6 \times 2 \mathrm{~mm}^{3}$ ) rectangular samples by SQUID magnetometery (Quantum Design MPMS) at $20 \mathrm{~K}$ in the magnetic fields up to $7 \mathrm{~T}$. The $J_{c}$ has been derived from the width of the magnetization loops $(2 \mathrm{~m})$ using critical state formula [3]

$$
J_{c}=\frac{4 m}{a^{2} b c\left(1-\frac{a}{3 b}\right)},
$$

where $m$ is magnetic moment, $a, b$ and $c$ are width, thickness and length of the sample $(a<b)$, and the magnetic field is directed along $c$. The detailed maps of the grains has been obtained by polarized optical microscopy (POM) and electron backscatter diffraction (EBSD). An extensive atomic force microscopy (AFM) study has been carried out to clarify properties of grain boundaries. 


\section{RESULTS AND DISCUSSION}

It was early recognized that grain boundaries (GBs) are directly associated with pinning in $\mathrm{MgB}_{2}$. For example, Larbalestier et al. [4] pointed to similarity between the pinning force curves in $\mathrm{MgB}_{2}$ and $\mathrm{Nb}_{3} \mathrm{Sn}$, where pinning is known to be mainly of GB origin [5]. A correlation between $J_{c}$ and grain size (or the total length of GBs per unit area) has been observed in [3] and a model accounting for the effect of the grains was found to give a good description of the pinning force curves for bulk $\mathrm{MgB}_{2}$ [6]. Based on this, a deeper search for defects of crystal lattice responsible for the pinning could be reduced to such items as GBs as whole, GB meander steps, GB nanoinclusions and GB dislocations.

The AFM images show that meandering is not typical to $\mathrm{MgB}_{2}$ and the GBs are mainly nanoinclusions free. This is found for GBs of all misorientation angles after exploring them by AFM and using as a guideline EBSD maps. The remaining two options for pinning: GBs as whole and dislocations in them require more detailed consideration.

Two typical AFM pictures of $\mathrm{MgB}_{2}$ are shown in Fig. 1. GBs are mainly flat and bear features of hexagonal structure (Fig. 1a). It is possible to observe etching pits of dislocations and creation of GBs by nanoparticles (Fig. 1b). Thus, even if nanoparticle are not pinning centers, they are able to improve pinning by creating GBs.
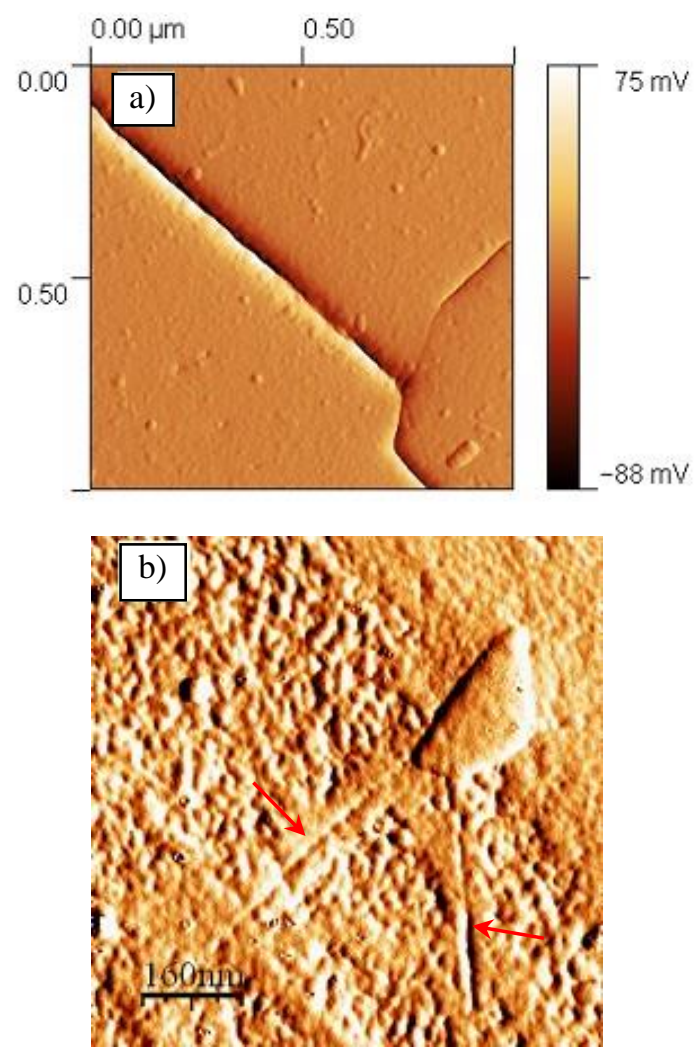

Fig. 1. Typical AFM pictures of dense $\mathrm{MgB}_{2}$. a) Flat GBs bearing features of hexagonal structure. b) Generation of GBs by a nanoparticle (shown by arrows). The etching dislocation pits are seen on the left GB.

By comparing POM and EBSD maps in $\mathrm{MgB}_{2}$ in [3], a large amount of high-angle twist GBs was found with rotations around the $c$-axis. Several of them are marked by dots in EBSD map in Fig. 2d. The number of misorientations as function of their angle for the GBs (shown separately with corresponding color in Fig. 2a) is plotted in Fig. 2b). The distinct peaks at misorientation angles of 28,53 and $70^{\circ}$ indicate that most of grain boundaries are low-energy coincident site lattice GBs [7].
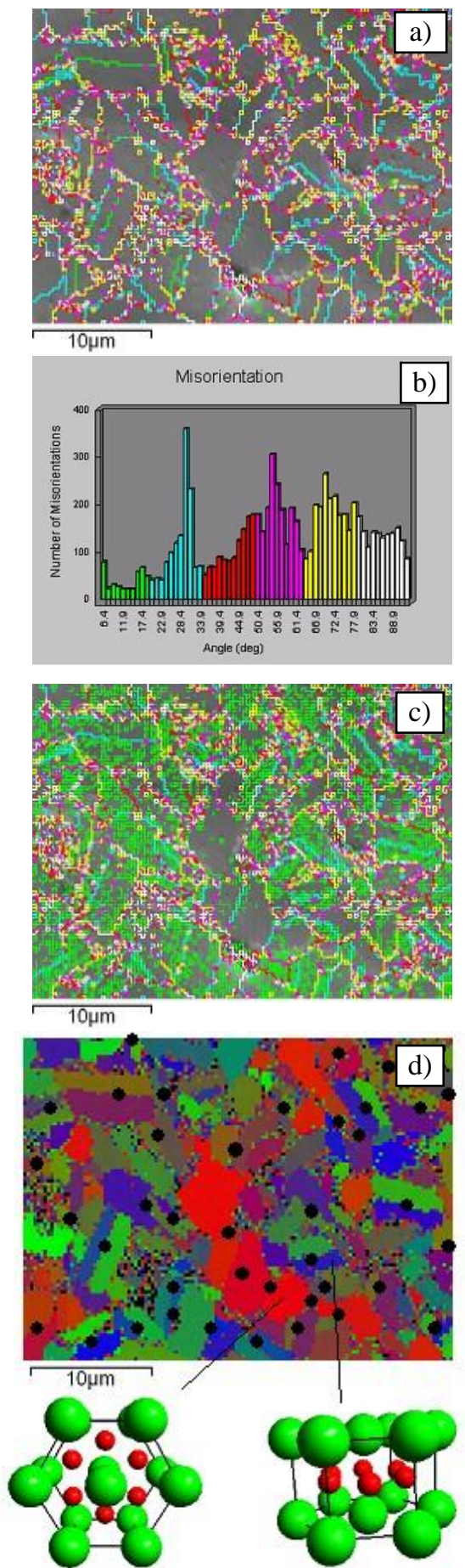

Fig. 2. a) EBSD GB map of a dense $\mathrm{MgB}_{2}$ with minimum misorientation angle of $5^{\circ}$. b) The number of misorientations as function of angle for the map shown in a) with colors corresponding to colors of GBs. c) GB map as in the plot a) at minimum misorientation angle of $0.8^{\circ}$. d) An EBSD grain map corresponding to GB maps in a) and c). The lower part of the plot shows direction of crystal lattice in selected parts of the sample. The areas, which are free from the low-angle boundaries in c) have c-axis directed perpendicular to the surface of the sample.

Figs. 2a),b),d) are obtained for the lowest detected misorientation angle of $5^{\circ}$. All misorientations below $5^{\circ}$ are not taken into account in them. The reduction of this angle to 
$0.8^{\circ}$ fills the GB map with low angle boundaries (green lines in Fig. 2c), but also introduces some noise. Surprisingly, some grains are not filled with low-angle GBs. The reason is evident after clarifying orientation of crystal lattice in all the grains. In the grains, which are free from the green lines, caxis is directed perpendicularly to the plane of the sample. No twist GBs with rotations around c-axis end on their surface. This indicates that most of the low-angle boundaries in c) are twist grain boundaries formed in the strained grains.

Many high-angle boundaries are also twist GBs. The domination of twist boundaries with rotations around c-axis in $\mathrm{MgB}_{2}$ is not a new result. It has already been reported in [8] and is a natural consequence of its layered structure of $\mathrm{MgB}_{2}$. When a set of growing $\mathrm{MgB}_{2}$ crystals with arbitrary directed c-axis is subjected to pressure, their accommodation into a dense structure follows a route with the lowest change in energy that mainly consists of the rotations of crystals and twists around the c-axis.

The GB maps and the angle $(\Theta)$ dependence of misorientations in Fig. 2 are ideal to derive the surface density of dislocations. To count dislocations, one can apply Frank's formula [9]:

$$
D=\frac{b / 2}{\sin (\Theta / 2)},
$$

where $D$ is the distance between dislocations and $\boldsymbol{b}$ is Burgers vector, which is the in-plane parameter of the lattice. The Eq. (2) is ideal for small $\Theta$ and tilt GBs that are rows of dislocations, but it also could be used for large $\Theta$. Using this equation, EBSD GB maps were processed for an intermediate minimum misorientation angle of $2^{\circ}$ in all 25 samples allowing calculating the surface density of dislocations $\left(D_{d}\right)$.

This resulted in Fig. 3, in which $J_{c}$ (derived from the magnetization loops at $20 \mathrm{~K}$ ) is plotted as function of $D_{d}$ at three magnetic fields of 0,2 and 4 Tesla. The black symbols show samples without nanoparticles and the red symbols with nanoparticles.

There are several important features in Fig. 3. First, $J_{c}\left(D_{d}\right)$ is different for different fields $(B)$. Second, samples with and without nanoparticles behave differently. At equal $D_{d}$ in lower fields, samples without nanoparticles have higher $J_{c}$ than samples with nanoparticles. Third, $J_{c}\left(D_{d}\right)$ is a non-linear function. Such a behavior is unexpected. If dislocations are pinning centers, $J_{c}\left(D_{d}\right)$ must be linear, as, for example, in $\mathrm{YBa}_{2} \mathrm{Cu}_{3} \mathrm{O}_{\mathrm{x}}$ films [10].

The variation of $J_{c}$ with $B$ is not surprising and reflects the limited number of pinning centers. The $B=0$ state is obtained by slow decrease of $B$ from a high value of few Tesla. The vortices are able to explore all pinning centers. Those trapped on the strongest pinning centers remain in the sample, while weakly-pinned exit. At $B=2 \mathrm{~T}$, the number of vortices is considerably higher than at $B=0$. All pinning sites, including weak ones, are filled. The critical current density drops because it is easy to displace weakly-pinned vortices. At $B=$ $4 \mathrm{~T}$, most of the vortices are weakly pinned and start moving at a very low current. Only the samples with largest $D_{d}$ can provide a considerable $J_{c}$. In this case, the $J_{c}\left(D_{d}\right)$ is an exponential function, and the advantage of nanoparticles becomes more obvious, Fig. $3 \mathrm{c}$.
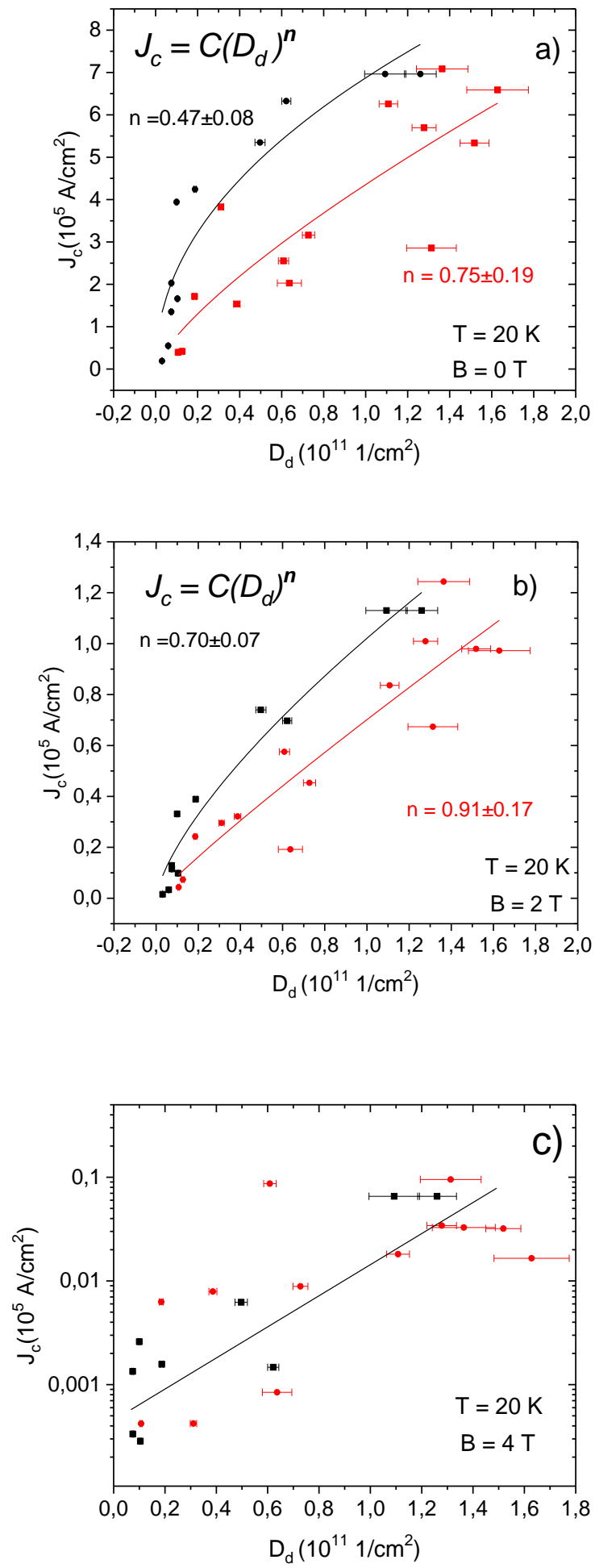

Fig. 3. Critical current density as function of the density of dislocations at $20 \mathrm{~K}$ and the magnetic field of 0 (a), $2 \mathrm{~T}$ (b) and $4 \mathrm{~T}$ (c). Black and red symbols represent samples without and with nanoparticles, respectively. The values of power exponent for $J_{c}\left(D_{d}\right)$ fitted with the Eq. (3) are shown in (a) and (b) for both groups of the samples.

In Figs. 3a),b), $J_{c}\left(D_{d}\right)$ is fitted with a function:

$$
J_{c}=C\left(D_{d}\right)^{n},
$$


where $C$ is a constant. The values of the power exponent $n$ are shown in the plots. For the samples with nanoparticles at $2 \mathrm{~T}, n$ is close to 1 and for those without nanoparticles, it is $0.7 \pm 0.07$. It is remarkable that for the samples without nanoparticles at zero field, $n$ becomes close to 0.5 .

There is simple explanation of this value of $n$. If dislocations are far from each other, the strained areas around them are not overlapping, and the order parameter of superconductor is suppressed only locally, what is ideal for pinning of isolated flux quanta. In this case, the coefficient $C$ in (3) is independent of density of dislocations, and the power exponent $n$ is equal to 1 . In contrast, when dislocations are close to each other, the strained areas overlap and the order parameter of superconductor is strongly suppressed reducing the pinning force. In addition, it becomes possible for vortices to slide along the rows of dislocations. This makes coefficient $C$ in (3) dependent on the density of dislocations. A realistic dependence is inverse proportionality to the distance between the dislocations, which, at homogeneous distribution of GBs, is proportional to square root of $D_{d}$. As a result, $J_{c}$ is also proportional to the square root of $D_{d}$. The presence of nanoparticles modifies Eq. (3) moving it closer to linear dependence on $D_{d}$. The dependence is also modified when density of magnetic flux becomes larger (see Fig. 3b)).

One of the differences between nanoparticles-free and nanoparticles-added samples is dominant type of GBs. The nanoparticles-free samples are mainly filled with twist grain boundaries (see Fig. 2). The nanoparticles usually create several GBs (Fig. 1), and not all of them are twist boundaries. Thus, nanoparticles-added samples are populated with tilt boundaries or GBs of a mixed tilt/twist origin too. For the clean tilt GBs, count of dislocations with Eq. (2) is exact and these are expected to give linear $J_{c}\left(D_{d}\right)$. The twist boundaries with rotations around $c$-axis can be represented by a network of crossed dislocations [7]. The flux quanta can be pinned either on these dislocations, or on their cross-points.

In a polycrystal with randomly oriented grains, vortex in any plane perpendicular to the applied magnetic field would find the closest cross-point and be pinned on it without changing much length and energy, moving from one plane to another. Based on the estimation of average grain size in [3], a realistic distance between cross-points of dislocations in twist grain boundaries in the samples with highest $J_{c}$ is 20-30 $\mathrm{nm}$. Thus, vortex would simultaneously be pinned on a very large number of cross-points. Naturally, these cross-points keep vortex from sliding along the grain boundaries.

Such a model is able to explain most features of Fig. 3. Twist boundaries are more effective than tilt or mixed boundaries due to large number of cross-points of dislocations. That is why in low fields samples without nanoparticles perform better than samples with nanoparticles. With the increase of field, more vortices become trapped on the tilt or mixed boundaries and $n$, therefore, moves from 0.5 to 1 in both groups of the samples.

The analysis in Fig. 3 is made for minimum misorientation angle of $2^{\circ}$. The decrease of the angle allows to detect more low-angle boundaries and dislocations inside the grains. However, due to the specific form of Eq. (2), their density for low angles is small and does not contribute much to the pinning. The decrease of $\Theta$ from 2 to $0.1^{\circ}$, for example, adds about $6 \%$ of dislocations only.

The remaining question about the interaction of vortex with the GB as a whole could be relevant if the vortex is parallel to GB, which is a very rare case in the system of large number of randomly-oriented grains.

In conclusion, using a range of dense $\mathrm{MgB}_{2}$ samples, it is found that critical current density follows a power-law dependence on the density of dislocations with the power exponent at low fields close to 0.5 for the samples without nanoparticles. It was also shown that vortices are mainly pinned on dislocations or their cross-points.

\section{REFERENCES}

[1] P. Mikheenko, "Possible superconductivity in the brain," J. Supercond. Novel Magn., https://doi.org/10.1007/s10948-018-4965-4, December 2018.

[2] S. X. Dou et al., "Mechanism of enhancement in electromagnetic properties of $\mathrm{MgB}_{2}$ by nano SiC doping," Phys. Rev. Lett., vol. 98, 097002, February 2007.

[3] P. Mikheenko, E. Martínez, A. Bevan, J. S. Abell, and J. L. MacManusDriscoll, "Grain boundaries and pinning in bulk $\mathrm{MgB}_{2}$," Supercond. Sci. Technol., vol. 20, pp. S264-S270, August 2007.

[4] D. C. Larbalestier et al., "Strongly linked current flow in polycrystalline forms of the superconductor $\mathrm{MgB}_{2}$," Nature, vol. 410, pp. 186-189, March 2001

[5] D. Dew-Hughes, "The role of grain boundaries in determining $J_{c}$ in high-field high-current superconductors," Phil. Mag., vol. 55, pp. 459479, April 1987.

[6] E. Martínez, P. Mikheenko, M. Martínez-López, A. Millán, A. Bevan, and J. S. Abell, "Flux pinning force in bulk $\mathrm{MgB}_{2}$ with variable grain size," Phys. Rev. B, vol. 75, 134515, April 2007.

[7] W. Bollmann, "Crystal Defects and Crystalline Interfaces," Berlin: Springer, 1972, $254 \mathrm{p}$.

[8] Y. Zhu et al., "Microstructure and structural defects in $\mathrm{MgB}_{2}$ superconductor," Physica C, vol. 356, pp. 239-253, August 2001.

[9] F. C. Frank, "The resultant content of dislocations in an arbitrary intercrystalline boundary",Symposium on the Plastic Deformation of Crystalline Solids, (ONR Pittsburgh), p. 150, May 1950.

[10] B. Dam et al., "Origin of high critical currents in $\mathrm{YBa}_{2} \mathrm{Cu}_{3} \mathrm{O}_{7-\delta}$ superconducting thin films," Nature, vol. 399, pp. 439-442, June 1999. 